\title{
Correlation of Immune-Related Adverse Events and Effects of Pembrolizumab Monotherapy in Patients with Non-Small Cell Lung Cancer
}

This article was published in the following Dove Press journal: Lung Cancer: Targets and Therapy

\section{Susumu Noguchi (D) \\ Keiichiro Suminaga \\ Takahiro Kaki \\ Hiroaki Kawachi \\ Akari Fukao \\ Satoshi Terashita (D) \\ Sadao Horikawa \\ Tatsuyoshi Ikeue \\ Takakazu Sugita}

Respiratory Medicine, Japanese Red Cross Wakayama Medical Center,

Wakayama, Japan
Correspondence: Susumu Noguchi Respiratory Medicine, Japanese Red Cross Wakayama Medical Center, 20-4, Komatsubara Street, Wakayama City 6408558, Japan

Tel $+8|-73-422-4| 7 \mid$

Fax +8I-73-426-II68

Emailsnoguchi515@gmail.com
Purpose: The effects of immune checkpoint inhibitors have been reported to be linked with immune-related adverse events (irAEs). In patients with advanced non-small-cell lung cancer, who tested positive for programmed death-ligand 1 (PD-L1), pembrolizumab, an immune checkpoint inhibitor can be used as a treatment, and it was found to improve overall survival. However, there are only a few reports on the relationship between the therapeutic effects of pembrolizumab in patients with lung cancer and the irAEs of pembrolizumab. The purpose of this study was to determine the correlation between immune-related adverse events and the effects of pembrolizumab monotherapy in patients with non-small-cell lung cancer.

Patients and Methods: From February 2017 to August 2019, we conducted a retrospective analysis of the effects of pembrolizumab treatment and immune-related adverse events in 94 patients with non-small-cell lung cancer treated with pembrolizumab only.

Results: In 63 cases, irAEs were observed. The most common irAE was rash. PD-L1 positivity $\geq 50 \%$ tended to cause irAEs. The median progression-free survival (PFS) rates with and without irAEs were 371 days (95\% CI, 184-NR) and 67 days (95\% CI, 51-87 days), respectively. In a multivariate analysis, irAEs and Eastern Cooperative Oncology Group performance status (PS) were the factors related to PFS.

Conclusion: In patients with lung cancer, who were treated with pembrolizumab monotherapy, the development of irAEs was likely indicative of the positive effects of pembrolizumab. This novel finding appears to be useful for clinicians who work with pembrolizumab for lung cancer treatment.

Keywords: lung cancer, pembrolizumab, adverse drug event, correlation study

\section{Introduction}

Immune checkpoint inhibitors (ICIs) have been approved as therapeutic treatments for a variety of carcinomas, and nivolumab has been approved for the treatment of non-small-cell lung cancer since 2015. In addition, the use of pembrolizumab has been approved since 2016, and it can be used as the primary treatment in programmed death-ligand 1 (PD-L1)-positive patients. Currently, pembrolizumab is one of the main treatments for patients with non-small-cell lung cancer. In particular, pembrolizumab is considered to be highly effective for patients with PD-L1 positivity $\geq 50 \%$. $^{1,2}$ The effects of ICIs have been reported to be associated with unique adverse events termed immune-related adverse events (irAEs), which are different from the adverse events of conventional anticancer agents. Although there 
are reports of irAEs in patients with non-small-cell lung cancer being treated with nivolumab, ${ }^{3-5}$ few reports also exist on the association between pembrolizumab treatment and irAEs in patients with non-small-cell lung cancer. In this study, we retrospectively examined the correlation of irAEs and the effects of pembrolizumab monotherapy in patients with non-small-cell lung cancer.

\section{Patients and Methods}

\section{Patients and Assessment}

From February 2017 to August 2019, data for 94 patients with non-small-cell lung cancer who were treated pembrolizumab only at the Japanese Red Cross Wakayama Medical Center were analyzed retrospectively. Treatment responses were evaluated using the Response Evaluation Criteria for Solid Tumors (RECIST) v1.1. PFS was calculated as time from pembrolizumab initiation until tumor progression as determined by attending physician, death from any cause, or last follow-up. For toxicity assessment, the Common Terminology Criteria for Adverse Events (CTCAE) v4.0 was used. Similar to that in previous reports, irAEs were defined as "adverse events with a potential immunologic cause that need frequent monitoring and potential intervention with immune suppression and/or endocrine treatment". 6 This study protocol was approved by the Japanese Red Cross Society Wakayama Medical Center ethics committee. This investigation was conducted according to the principles expressed in the Declaration of Helsinki. Since this study was conducted using only existing information, no written or verbal consent was obtained from patients. However, the information about the research was published to patients (posted in the hospital), and patients were given the opportunity to refuse to carry out the research via the opt-out system.

\section{Statistical Analysis}

The clinical and demographic characteristics of the patients were compared using Fisher's exact test. The Kaplan-Meier method and Log-rank test were used to evaluate progression-free survival (PFS). Landmark analysis at 9 weeks was also performed (irAEs $n=41$, NoirAEs $n=33$ ) because it was the most suitable time for judging the first effect. To evaluate the effect of irAEs on PFS, Cox proportional hazards regression analysis was performed. Statistical significance was defined as a
$\mathrm{P}$ value $<0.05$, and all analyses were performed using $R$ version 1.40 .

\section{Results}

Of the 94 patients in total, 63 (67\%) developed irAEs. The observed irAEs and frequency are shown in Table 1. The most common irAE was a rash, followed by interstitial pneumonia. With regard to eosinophilia, the evaluation was made on the item of Investigations-Other, specify of CTCAE v4.0. It was assumed that the blood sampling test showed a prolongation of 2 or more times after the clear rise from the base line.

The patients' backgrounds according to the presence or absence of irAEs are shown in Table 2. PS0-1 and PD-L1 positivity $\geq 50 \%$ was correlated with an increased number of irAEs.

The median duration of follow-up was 282 days (4-856 days). At the time of last follow-up, 61 patients $(64.9 \%)$ were alive and 27 patients $(28.7 \%)$ had been treated with pembrolizumab. The median PFS and OS for the whole cohort were 159 days (95\% CI, 91-335 days) and NR (421-NR).

The median PFS rates with and without irAEs were 371 days (95\% CI, 184-NR days) and 67 days (95\% CI, 51-87 days), respectively. PFS was significantly prolonged in the group that developed irAEs (hazard ratio [HR], 0.2376; 95\% CI, 0.1348-0.4187; $P<0.0001$ ) (Figure 1). Although the difference was not significant, the landmark analysis at 9 weeks indicated prolonged PFS and OS in patients who developed irAEs than in those who did not. The median PFS rates with and without irAEs were 335 days (95\% CI, 184-NR days) and 201 days (95\% CI, 112-NR days), respectively. $(P=0.321)$ (Figure 2). The median OS with and

Table I Distribution of Immune-Related Adverse Events

\begin{tabular}{|l|l|l|l|}
\hline $\begin{array}{l}\text { Immune Related Adverse } \\
\text { Event }\end{array}$ & $\begin{array}{l}\text { Grade } \\
\text { I }\end{array}$ & $\begin{array}{l}\text { Grade } \\
\mathbf{2}\end{array}$ & \begin{tabular}{l} 
Grade $\mathbf{3}$ or \\
\hline Rash
\end{tabular} \\
& 10 & $\mathrm{II}$ & $5(5.3)$ \\
Pneumonitis & $(10.6)$ & $(\mathrm{II} .7)$ & \\
Fever & $6(6.4)$ & $6(6.4)$ & $\mathrm{I}(\mathrm{I} . \mathrm{I})$ \\
Thyroid dysfunction & $9(9.6)$ & 0 & 0 \\
Colitis & $3(3.2)$ & $4(4.3)$ & 0 \\
Eosinophilia & 0 & $4(4.3)$ & $2(2.1)$ \\
Hepatic dysfunction & $4(4.3)$ & 0 & 0 \\
Adrenal insufficiency & $3(3.2)$ & 0 & 0 \\
Infusion reaction & 0 & 0 & $2(2.1)$ \\
\hline
\end{tabular}


Table 2 Demographics of 94 Patients Stratified by History of Ir AEs

\begin{tabular}{|c|c|c|c|c|}
\hline Characteristic & $\begin{array}{l}\text { All } \\
(n=94)\end{array}$ & ir $\operatorname{AEs}(+)(n=63)$ & ir $\operatorname{AEs}(-)(n=3 I)$ & $P$ value \\
\hline Age & $70(37-86)$ & $70(37-86)$ & $68(46-83)$ & 0.452 \\
\hline Number of doses & $5(|-3|)$ & $6(|-3|)$ & $3(1-15)$ & 0.001 \\
\hline \multicolumn{5}{|l|}{ Sex, n (\%) } \\
\hline Male & $82(87.2)$ & $57(90.5)$ & $25(80.6)$ & 0.201 \\
\hline Female & $12(12.8)$ & $6(9.5)$ & $6(19.4)$ & \\
\hline \multicolumn{5}{|l|}{ Smoking, n (\%) } \\
\hline Ever & $84(89.4)$ & $58(92.1)$ & $26(83.9)$ & 0.289 \\
\hline Never & $10(10.6)$ & $5(7.9)$ & $5(16.1)$ & \\
\hline \multicolumn{5}{|l|}{ ECOG PS, n (\%) } \\
\hline $0-1$ & $83(88.3)$ & $60(95.2)$ & $23(74.2)$ & 0.005 \\
\hline$\geqq 2$ & II (II.7) & $3(4.8)$ & $8(25.8)$ & \\
\hline \multicolumn{5}{|l|}{ PD-LI expression, $n$ (\%) } \\
\hline$\geqq 50 \%$ & 71 (75.5) & $54(85.7)$ & $17(54.8)$ & 0.002 \\
\hline $\mathrm{I}-49 \%$ & $23(24.5)$ & $9(14.3)$ & 14 (45.2) & \\
\hline \multicolumn{5}{|l|}{ Treatment line, $n(\%)$} \\
\hline First line & $40(42.6)$ & $30(47.6)$ & $10(32.3)$ & 0.187 \\
\hline$\geqq$ Second line & $54(57.4)$ & $33(52.4)$ & $21(67.7)$ & \\
\hline \multicolumn{5}{|l|}{ Histology, n (\%) } \\
\hline NonSq & $71(75.6)$ & $48(76.2)$ & $23(74.2)$ & 1.00 \\
\hline $\mathrm{Sq}$ & $23(24.4)$ & $15(23.8)$ & $8(25.8)$ & \\
\hline
\end{tabular}

Abbreviations: irAEs, immune-related adverse events; ECOG PS, Eastern Cooperative Oncology Group performance status; PD-LI, programmed death ligand I: Sq, squamous cell carcinoma.

without irAEs were NR (95\% CI, 421-NR) and NR (95\% CI, 564-NR) $(P=0.054)$ (Figure 3). On multivariate analysis, irAEs and PS were found to be associated with prolonged PFS (Table 3).

\section{Discussion}

We retrospectively examined 94 patients treated with pembrolizumab only. Previously, it was reported that ICIs are more effective when accompanied by irAEs occurs. ${ }^{7,8}$ Pembrolizumab has been reported to be more effective when dermatosis develops in patients with melanoma. ${ }^{9,10}$ Lisberg et al reviewed the KEYNOTE001 trial at their own facility and reported that the positive effects of pembrolizumab could be expected if an adverse event occurred. ${ }^{11}$ Consistent with their findings, our report confirmed that pembrolizumab can be effective even if an adverse event occurs and that the effect may be diminished at PS2 or higher. In contrast, in a retrospective study using pembrolizumab to treat patients with non-small-cell lung cancer, Ksienski et al reported that there was no association between the onset of irAEs and overall survival. ${ }^{12}$ A recent study reported that there was a possibility that specific autoimmune T-cell clones recognize shared self-antigens of lung cancer and the skin, thereby causing patients who experience good therapeutic effects against lung cancer to develop skin disorders. ${ }^{13}$ In our study, rashes were found to be the most common irAE, and it is possible that this reflects the therapeutic effect of pembrolizumab.

Pembrolizumab is highly effective in patients with PD-L1 positivity $\geq 50 \%$. In this study, we showed that patients who developed irAEs had significantly increased PFS. Even in the landmark analysis, although the difference was not significant, PFS tended to be longer in those who developed irAEs than in those who did not. PD-L1 positivity $\geq 50 \%$ tended to be correlated better with irAEs. Further, multivariate analysis suggested that irAEs are the factors associated with prolonged PFS. These results suggest that the onset of 


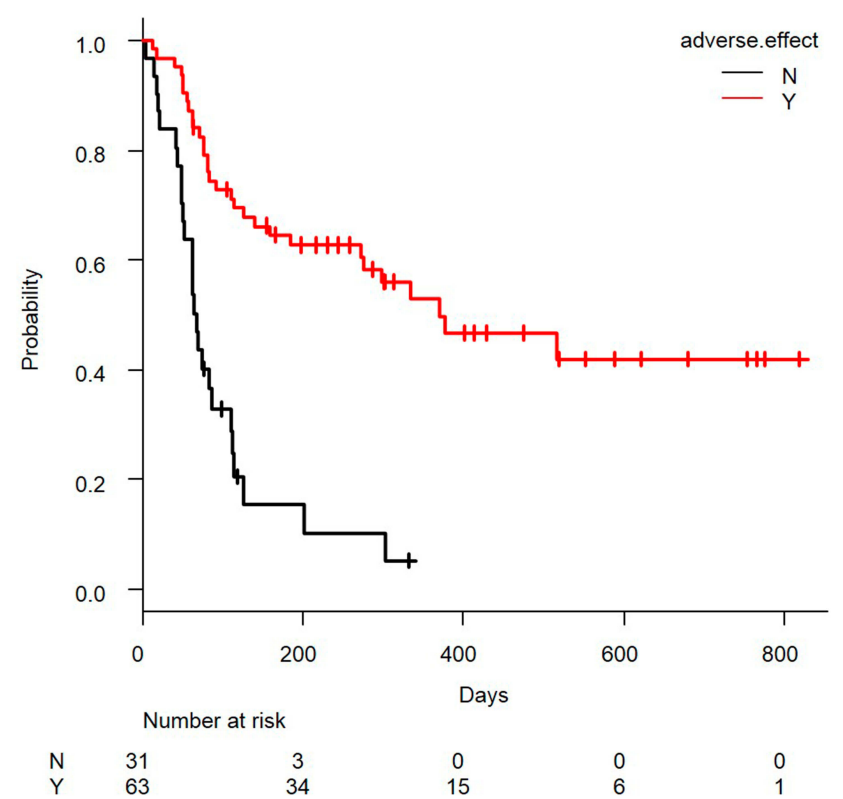

Figure I Kaplan-Meier curve of progression-free survival (red line, irAEs group; black line, no-irAEs group), $\mathrm{Y}=\operatorname{irAEs}(+), \mathrm{N}=\operatorname{irAEs}(-)$.

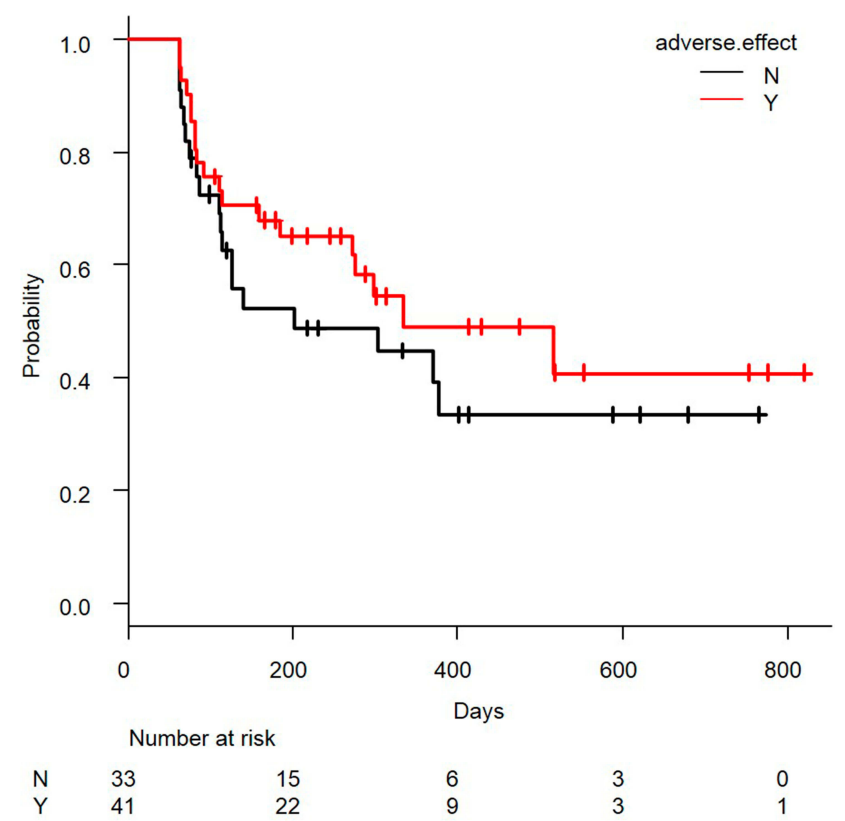

Figure 2 Kaplan-Meier curve of progression-free survival with 9-week landmark analysis (red line, irAEs group; black line, no-irAEs group).

irAEs may be a predictor of efficacy in addition to PDL1 positivity $\geq 50 \%$.

With respect to PS, there have been reports that the effect of pembrolizumab decreases at $\mathrm{PS} \geq 2,{ }^{14,15}$ which is consistent with the results of our study. However, of the 94 cases only $11(11.7 \%)$ had PS $\geq 2$, and accumulation of additional data on this seems necessary.

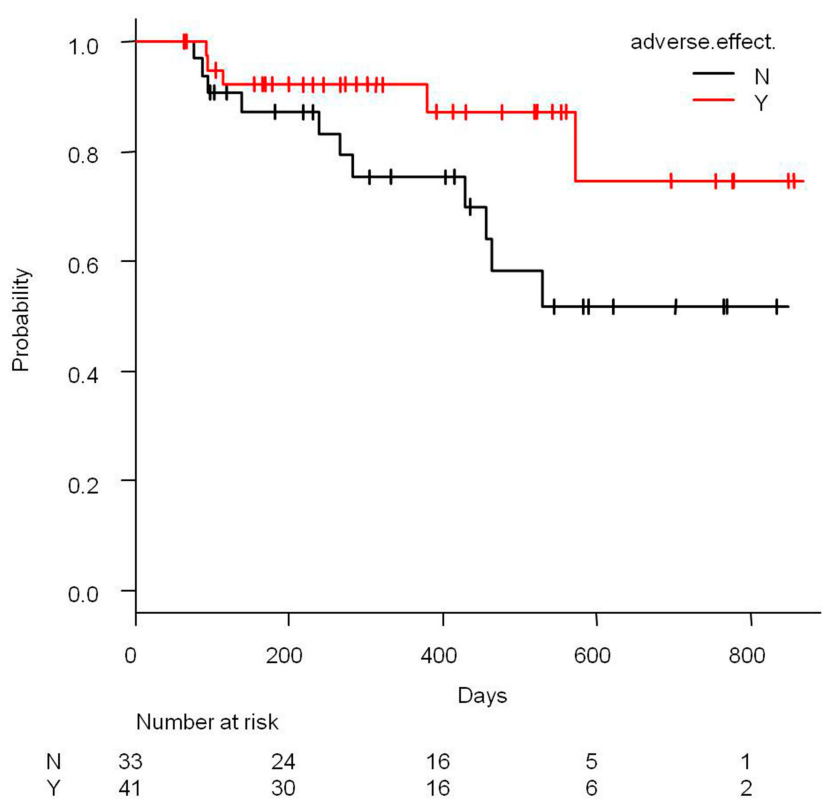

Figure 3 Kaplan-Meier curve of overall survival with 9-week landmark analysis (red line, irAEs group; black line, no-irAEs group).

Our study has limitations. This was a single-center retrospective study; therefore, there is a possibility of bias in the analysis. However, with regard to the evaluation of side effects, nurses and pharmacists describe it in the medical chart independently, which increases the accuracy of the evaluation. Next, the number of cases was limited, and we think that an accumulation of additional cases to substantiate our findings is necessary in the future.

\section{Conclusion}

In conclusion, in patients with lung cancer being treated with pembrolizumab the development of irAEs may be indicative of favorable effects as a consequence of pembrolizumab treatment. Although additional cases are needed to substantiate our findings, our results may be useful in determining the efficacy of pembrolizumab treatment in patients with lung cancer.

Table 3 Multivariate Cox Proportional Hazard Regression Analysis on PFS

\begin{tabular}{|l|l|l|}
\hline Variable & HR $(\mathbf{9 5} \% \mathbf{~ C I})$ & P value \\
\hline Ir AEs & $0.33(0.17-0.65)$ & $0.00 I$ \\
PD-LI $\geqq 50 \%$ & $0.80(0.39-I .64)$ & 0.550 \\
PSO-I & $0.20(0.09-0.44)$ & $<0.00 I$ \\
Treatment line(I vs $\geqq 2)$ & $1.28(0.7 I-2.3 I)$ & 0.419 \\
Brain/liver meta & $I .47(0.76-2.86)$ & 0.255 \\
Sex & $0.69(0.33-I .42)$ & 0.314 \\
\hline
\end{tabular}

Abbreviations: PFS, progression-free survival; irAEs, immune-related adverse events; PD-LI, programmed death ligand I; PS, performance status. 


\section{Abbreviations}

irAEs, immune-related adverse events; ICI, immune checkpoint inhibitor; PD-L1, programmed death-ligand 1; PFS, progression-free survival; PS, performance status.

\section{Acknowledgment}

We would like to thank Keita Hirano, MD, of the Department of Nephrology, Kyoto University Hospital for advice on the statistical analysis.

\section{Disclosure}

The authors have not received any funding for this study and report no conflicts of interest in this work.

\section{References}

1. Reck M, Rodríguez-Abreu D, Robinson AG, et al. Pembrolizumab versus chemotherapy for PD-L1-positive non-small-cell lung cancer. $N$ Eng $J$ Med. 2016;375(19):1823-1833. doi:10.1056/NEJMoa1606774

2. Mok TSK, Wu YL, Kudaba L, et al. Pembrolizumab versus chemotherapy for previously untreated, PD-L1-expressing, locally advanced or metastatic non-small-cell lung cancer (KEYNOTE-042): a randomized, open-label, controlled, Phase 3 trial. Lancet. 2019;393:1819-1830. doi:10.1016/S0140-6736(18)32409-7

3. Haratani K, Hayashi H, Chiba Y, et al. Association of immune-related adverse events with nivolumab efficacy in non-small-cell lung cancer JAMA Oncol. 2018;4:374-378. doi:10.1001/jamaoncol.2017.2925

4. Teraoka S, Fujimoto D, Morimoto T, et al. Early immune-related adverse events and association with outcome in advanced non-small cell lung cancer patients treated with nivolumab: a prospective cohort study. J Thorac Oncol. 2017;12:1798-1805.

5. Sato K, Akamatsu H, Murakami E, et al. Correlation between immunerelated adverse events and efficacy in non-small cell lung cancer treated with nivolumab. Lung Cancer. 2018;115:71-74. doi:10.1016/ j.lungcan.2017.11.019
6. Weber JS, Hodi FS, Wolchok JD, et al. Safety profile of nivolumab monotherapy: a pooled analysis of patients with advanced melanoma. J Clin Oncol. 2017;35(7):785-792. doi:10.1200/JCO.2015.66.1389

7. Shafgat H, Gourdin T, Sion A. Immune-related adverse events are linked with improved progression-free survival in patients receiving anti-PD-1/PD-L1 therapy. Semin Oncol. 2018;45(3):156-163. doi:10.1053/j.seminoncol.2018.07.003

8. Sakakida T, Ishikawa T, Uchino J, et al. Clinical features of immunerelated thyroid dysfunction and its association with outcomes in patients with advanced malignancies treated by PD-1 blockade. Oncol Lett. 2019;18:2140-2147. doi:10.3892/ol.2019.10466

9. Sanlorenzo M, Vujic I, Daud A, et al. Pembrolizumab cutaneous adverse events and their association with disease progression. JAMA Dermatol. 2015;151(11):1206-1212. doi:10.1001/jamadermatol.2015.1916

10. Hua C, Boussemart L, Mateus C, et al. Association of vitiligo with tumor response in patients with metastatic melanoma treated with pembrolizumab. JAMA Dermatol. 2016;152:45-51. doi:10.1001/ jamadermatol.2015.2707

11. Lisberg A, Tucker DA, Goldman JW, et al. Treatment-related adverse events predict improved clinical outcome in NSCLC patients on KEYNOTE-001 at a single center. Cancer Immunol Res. 2018;6 (3):288-294. doi:10.1158/2326-6066.CIR-17-0063

12. Ksienski D, Wai ES, Croteau N, et al. Pembrolizumab for advanced nonsmall-cell lung cancer: efficacy and safety in everyday clinical practice. Lung Cancer. 2019;133:110-116. doi:10.1016/j.lungcan.2019.05.005

13. Berner F, Bomze D, Diem S, et al. Association of checkpoint inhibitor-induced toxic effects with shared cancer and tissue antigens in non-small cell lung cancer. JAMA Oncol. 2019;5(7):1043-1047. doi:10.1001/jamaoncol.2019.0402

14. Ksienski D, Wai ES, Croteau N, et al. Efficacy of nivolumab and pembrolizumab in patients with advanced non-small-cell lung cancer needing treatment interruption because of adverse events: a retrospective multicenter analysis. Clin Lung Cancer. 2019;20(1):e97e106. doi:10.1016/j.cllc.2018.09.005

15. Tamiya M, Tamiya A, Hosoya K, et al. Efficacy and safety of pembrolizumab as first-line therapy in advanced non-small cell lung cancer with at least 50\% PD-L1 positivity: a multicenter retrospective cohort study (HOPE-001). Invest New Drugs. 2019;37(6):1266-1273. doi:10.1007/s10637-019-00843-y

\section{Publish your work in this journal}

Lung Cancer: Targets and Therapy is an international, peerreviewed, open access journal focusing on lung cancer research, identification of therapeutic targets and the optimal use of preventative and integrated treatment interventions to achieve improved outcomes, enhanced survival and quality of life for the cancer patient. Specific topics covered in the journal include: Epidemiology, detection and screening; Cellular research and biomarkers; Identification of biotargets and agents with novel mechanisms of action; Optimal clinical use of existing anticancer agents, including combination therapies; Radiation and surgery; Palliative care; Patient adherence, quality of life, satisfaction; Health economic evaluations. 